\title{
SY12-1 Symposium
}

\section{Neonatal Clinical Pharmacology}

John N. Van Den Anker ${ }^{1,2,3}$

${ }^{1}$ University of Basel Children's Hospital, Switzerland and Children's National Health System, Washington, DC, USA, ${ }^{2}$ University Children's Hospital Basel, Switzerland, ${ }^{3}$ Erasmus Medical Center-Sophia Children's Hospital, the Netherlands

The clinical outcome of preterm and term neonates can be significantly improved with the use of safe and effective medicines appropriately investigated in this patient population. Neonates are not just little infants or children. They need a much more detailed approach in decisions about when to treat a disease or condition, with which medicine and most importantly with what kind of dosing regimen for that particular medicine. However, even in 2018, health care professionals involved in neonatal care are routinely prescribing drug formulations, and applying dosing regimens, that initially have been developed for use in adults. Furthermore, the medicines these professionals are using were developed with adult pathophysiology in mind, and are based on adult indications. The overall aim of administering a specific drug to a patient is to reach effective treatment for a given disease while avoiding disproportional side-effects. Neonatal clinical pharmacology aims to predict drug-specific (side)-effects based on its pharmacokinetics and pharmacodynamics. Pharmacokinetics (PK, absorption, distribution and elimination, through either metabolism or primary renal elimination, ADME) hereby estimates the relationship between a drug concentration at a specific site (e.g. plasma, cerebrospinal fluid) and time after its administration (what the body does to the drug). Pharmacodynamics (PD) aims at both the effects and side-effects of a given drug (what the drug does to the body). PK and PD display extensive variability with population specific characteristics because of the fast maturational changes in the neonatal period and early infancy, while side effects may be more difficult to detect, both in the short-term but especially in the long-term. This presentation will give practical examples of the most recent developments in the area of neonatal clinical pharmacology. 